\title{
Influence des performances zootechniques sur les caractéristiques des tissus adipeux d'agneaux de bergerie, sevrés précocement et alimentés avec un régime à forte concentration énergétique
}

\author{
G Bozzolo 1*, M Bouillier-Oudot 1, E de Boisseson 1, \\ $M$ Ghassan 1, D Grasset 2 \\ ${ }^{1}$ ENSAT, laboratoire de zootechnie, 31076 Toulouse Cedex; \\ 2 GEBRO, 12100 Millau, France
}

(Reçu le 23 avril 1990; accepté le 15 juin 1990)

\begin{abstract}
Résumé - Les relations entre les performances zootechniques et l'apparition des gras huileux et brun-jaunâtre ont été étudiées sur des agneaux Lacaunes sevrés, des 2 sexes, alimentés avec un régime à forte concentration énergétique $(2,9 \mathrm{Mcal}$ (EM) $/ \mathrm{kg}$ (MS), sous forme de granulés distribués à volonté. L'échantillon a été constitué à partir de 54 individus sélectionnés parmi 1137 animaux, en raison de leur situation dans des classes extrêmes de tenue de carcasse après $18 \mathrm{~h}$ de ressuage en chambre frigorifique ( $15 q$ et 150 à gras mous; $14 \%$ et 100 à gras fermes). Sevrés à $32 \mathrm{j}$ en moyenne, ceux-ci furent abattus à partir d'un poids vif de $30 \mathrm{~kg}$ pour les femelles et de $39 \mathrm{~kg}$ pour les mâles. Les sujets ayant bénéficié d'un moindre allaitement maternel et accusant une forte vitesse de croissance (gmq ơ: $368 \mathrm{~g}$ contre $324 \mathrm{~g} ; \mathrm{gmq}$ ○: $307 \mathrm{~g}$ contre $264 \mathrm{~g}$ ), notamment chez les mâles, manifestent plus fréquemment ces défauts de carcasse. Corrélativement la diminution de l'âge à l'abattage ( $\sigma^{\prime: 111 ~ j}$ contre $123 \mathrm{j} ; \mathrm{Q}: 97 \mathrm{j}$ contre $113 \mathrm{j}$ ) et les faibles durées d'engraissement ( $0^{\prime}: 80$ contre 88 j; $9: 66$ contre 78 j) provoquent l'apparition des graisses molles, souvent colorées. La composition du tissu gras, semblable pour les 2 sexes, fait ressortir une plus forte teneur en eau pour les gras huileux $(26 \%)$ et une représentation majorée des proportions d'acides gras monoinsaturés à chaîne moyenne : $\Sigma C_{12: 1}$ à $C_{17: 1}(+112 \%)$ et, secondairement des acides gras ramifiés et impairs. Les gras durs et blancs, en plus d'une moindre teneur en eau (15\%) présentent une proportion élevée de $\mathrm{C}_{18: 0}(+104 \%)$ et de $\mathrm{C}_{16: 0}(+32 \%)$. Les autres acides gras n'apparaissent pas influencer de manière significative la tenue de carcasse. De même, l'analyse sérologique de différents paramètres : œstradiol, progestérone, testostérone, thyroxine, cortisol, vit $\mathrm{B}_{12}$, ne permet pas de faire apparaître des différences dans l'échantillon retenu.
\end{abstract}

gras / qualité / carcasse / agneau / ration

Summary - Effect of animal husbandry scores upon carcasses and fat characteristics of lambs weaned early and fed a high energy diet. The relation between husbandry scores and the apparition of soft and oily and yellow-brown fats were examined in Lacaune lambs. Fifty-four rams and ewes selected from 1137 animals were fed ad libitum a high metabolizable energy-pelleted diet $(2,9 \mathrm{Mcal}(E M) / \mathrm{kg} D M)$. Their carcasses were classified on opposite grades of firmness and whiteness after $18 \mathrm{~h}$ in a chilling room (15 $\$$ and $150^{\prime}$ with soft fat : grade $1 ; 14 \%$ and $100^{t}$ with firm fat : grade 5). Lambs weaned at an average of $32 d$ were slaughtered in 2 live weight groups at $39 \mathrm{~kg}$ for

* Correspondance et tirés à part 
the rams and at $30 \mathrm{~kg}$ for the ewes. The ones which received a shorter maternal alimentation ( $30 \mathrm{dvs}$ $35 \mathrm{~d}$ ) and a faster rate of growth ( of:368 $\mathrm{g} / \mathrm{d}$, vs $324 \mathrm{~g} / \mathrm{d}$; $9: 307 \mathrm{~g} / \mathrm{d}$, vs $264 \mathrm{~g} / \mathrm{d}$ ), in particular the rams, showed these carcass defects more frequently. Correlative shorter period of fattening ( $\sigma^{\prime}: 80 \mathrm{~d}$ vs $88 d ; 8: 66 d$ vs $78 d$ ) and younger time of slaughtering ( $d^{\prime: 111}$ vs $123 d$ old; $9: 97$ vs $113 d$ old) increased apparition of soft and colored subcutaneous fats. The composition of these fats is independent of the sex. It possesses a higher level of moisture (26\% vs $15 \%$ ) and higher level of medium chain unsaturated fatty acids: $\Sigma C_{12: 1}$ to $C_{17: 1}(+112 \%)$. There were consequently more odd numbered and branched chain fatty acids than firm carcasses. In contrast, firmer and whiter fats were higher in $C_{18: 0}(+104 \%)$ and $C_{16: 0}(+32 \%)$. In this study, neither other fatty acids nor several seric components such as estradiol and progesterone and testosterone and thyroxin and cortisol and vita$\min B_{12}$ had significant influence on the quality of carcasses. Some explanations are discussed. Adequate weaning and transition diet on the one hand and finition fatty diet on the other hand, should be based on the sex and genetic potential growth of individual lambs. This could be a method of achieving better quality in lamb carcasses.

fat / quality / carcass / lamb / diet

\section{INTRODUCTION}

Les agneaux Lacaune du rameau laitier, destinés à la production d'agneaux de boucherie, après un sevrage précoce (3 à 5 semaines), sont nourris intensivement avec un régime à base de concentrés. Ce type de spéculation génère des charges économiques importantes. Compte tenu de l'évolution des cours et des particularités des mécanismes communautaires de régulation du marché de la viande ovine, ces systèmes connaissent aujourd'hui, une certaine fragilité. La seule productivité pondérale n'est plus suffisante pour garantir le revenu; il importe d'assurer, en plus, la qualité du produit. Dès le premier niveau des transactions commerciales, les qualités de la carcasse constituent un prérequis déterminant. Aux références désormais classiques touchant à la conformation, à l'état d'engraissement (grille Europa), aux classes correspondant au poids des carcasses, s'ajoutent des notions liées à la présentation des carcasses réfrigérées. La tenue (fermeté et texture des gras), la couleur des gras externes et même de la viande, parfois les qualités organoleptiques sous-jacentes retiennent l'attention des professionnels.
En effet, lorsque les carcasses présentent des gras mous à texture huileuse et souvent de coloration brun-jaunâtre, attributs de l'agneau "bis", elles sont commercialement dépréciées. A la découpe, les pièces tirées de tels animaux ont une consistance molle, résultat d'un glissement des plans musculaires les uns par rapport aux autres (Aurousseau, 1981). Par ailleurs, des flaveurs prononcées, voire désagréables (Field et al, 1978; Aurousseau, 1981; Girard et al, 1986) sont associées à ce caractère. Enfin, la tendreté et la jutosité pourraient être indirectement influencées par l'orientation des métabolismes et l'évolution des différents constituants de la viande (Moody et al, 1980; Aurousseau, 1986; Girard et al, 1986; Solomon et Lynch, 1986; Vigneron et al, 1986).

De multiples facteurs incriminés dans l'apparition des gras mous ont été mis en évidence (Molénat et Thériez, 1973; Orskov et al, 1975; Miller et al, 1980; Aurousseau, 1981, 1986) notamment le degré d'insaturation élevé de leurs acides gras, associé à une forte teneur en eau (Paruelle et Pain, 1982; Miller et al, 1980). Mais les faits expérimentaux et les observations directes chez les producteurs révè- 
lent une forte variabilité dans la réponse des animaux, parfois des résultats opposés.

Parmi les facteurs d'élevage exerçant la plus forte incidence, le sexe des agneaux apparaît au premier plan : les femelles plus précoces, développent des gras souscutanés plus saturés (Molénat et Thériez, 1973) en même temps qu'un niveau d'engraissement supérieur (Crouse et al, 1978). Leur gras est souvent moins coloré.

Le degré de maturité tissulaire dépend de l'âge et, en interaction avec la précocité, conditionne la quantité et la composition des tissus adipeux. En conséquence, l'âge d'abattage retenu et/ou la précocité génétique des individus pourraient être à l'origine des écarts de tenue observés.

Mais ce sont surtout, pour des agneaux âgés de 3 mois, les causes alimentaires qui sont les plus évoquées (Garton et al, 1972; Ørskov et al, 1975; Field et al, 1978; Miller et al, 1980; Aurousseau, 1981; Busboom et al, 1981). Celles-ci sont liées à l'emploi de rations très énergétiques, riches en amidon, génératrices d'une orientation prononcée des fermentations dans le rumen de type propionique. La désaturation des acides gras longs en monoènes, au cours de la digestion, peut constituer une autre cause de l'apparition de gras mous (Toullec et Quillet, 1987). Enfin, des apports surabondants d'acide propionique, éventuellement aggravés par une carence relative en vitamine $B_{12}$, débordent les capacités hépatiques à transformer l'acide méthylmalonique en acide succinique. II en résulte une synthèse intense d'acides gras ramifiés et leur accumulation dans les tissus adipeux est une cause du manque de fermeté du tissu gras externe (Elliot, 1980; Aurousseau, 1986).

Pourtant dans le cas des travaux de Solomon et Lynch, 1988, en accord avec ceux de Crouse et al, 1978, les gras sous- cutanés les plus fermes et les plus blancs sont associés à un fort degré d'engraissement et sont obtenus avec le régime alimentaire le plus riche en énergie $(2,79$ Mcal (EM)/kg 1,87 Mcal (EM) $/ \mathrm{kg}$ ).

A partir des hypothèses formulées, diverses recommandations, tant de nature technique qu'alimentaire, ont été proposées: âge au sevrage, effet d'un rationnement, concentration énergétique et niveau azoté de la ration, introduction de foin de luzerne ou de pulpe de betterave, nature et conditionnement des céréales employées etc (Duncan et al, 1974; Ørskov et al, 1975; Van Quackebeke et al, 1978) sans permettre de maîtriser entièrement ce défaut de carcasse. On peut ainsi observer une variabilité importante dans la présentation des carcasses réfrigérées, en particulier avec des agneaux Lacaune sevrés et nourris avec un même aliment.

Une étude comparative, à partir d'agneaux extériorisant des profils de tenue de carcasses extrêmes nous semblait donc intéressante pour tenter d'affiner les liaisons entre les causalités d'ordre zootechnique (âge en début d'engraissement, vitesse de croissance, durée d'engraissement, sexe), d'ordre chimique sous l'angle des différences de composition des gras sous-cutanés - ou hormonal associé à la régulation de l'adipogénèse, pour éventuellement déboucher, à terme, sur des propositions d'élevage complémentaires.

\section{MATÉRIEL ET MÉTHODES}

\section{Les animaux}

Les agneaux de race Lacaune proviennent des séries de testage organisées dans les élevages laitiers affiliés au GEBRO (groupement des éleveurs du bassin de Roquefort). Ceux-ci sont 
issus d'un croisement : mâles de la souche lacaune-viande $x$ agnelles du rameau laitier. Ils sont sevrés à un âge compris entre 25 et $50 \mathrm{j}$, regroupés en bergerie et engraissés selon un protocole de conduite et d'alimentation identique.

Un échantillon de 54 agneaux a été retenu pour analyser l'influence des paramètres zootechniques. Trente agneaux ont servi aux comparaisons de la composition du gras de couverture, et des paramètres sériques.

Les aliments à densité énergétique élevée, conditionnés sous forme de granulés, sont offerts à volonté. Durant les 3 premières semaines, un aliment de démarrage (1) assure la transition après sevrage; par la suite un aliment unique (2) sert à l'engraissement jusqu'à son terme. Ceux-ci présentent les caractéristiques suivantes (tableau I).

Complémentairément, de la paille est mise à la libre disposition des agneaux.

Les animaux sont abattus au-delà de 60 jours d'enǵraissement, dès lors que mâles et femelles ont dépassé des poids vifs minima respectifs de 39 et $30 \mathrm{~kg}$ (impératif commercial).

\section{Paramètres zootechniques mesurés}

Le mode de naissance (Nais) se réfère à la taille de portée. Pendant la phase d'élevage, les paramètres zootechniques enregistrés concer-

Tableau 1. Composition des aliments fournis aux agneaux pendant la durée de l'expérience.

\section{Composition $/ \mathrm{kg} \quad$ Aliment 1 Aliment 2 d'aliment}

$\begin{array}{lrc}\text { Mat sèche } & 86,0 \% & 86,0 \% \\ \text { Mat cellulosique } & 7,0 \% & 5,0 \% \\ \text { Lignine } & 2,0 \% & 1,4 \% \\ \text { Mat minérale } & 7,5 \% & 8,0 \% \\ \text { Mat grasse } & 2,5 \% & 2,5 \% \\ \text { Mat protéique brute } & 16,0 \% & 14,5 \% \\ & & (2,6 \% \text { d'urée) } \\ \text { UFV } & 0,89 & 0,92 \\ \text { EM en Mcal } & 2,40 & 2,48\end{array}$

nent l'âge (Aent) et le poids (Pent) d'entrée des animaux en station d'engraissement, le poids après 60 j d'engraissement $\left(P e_{60}\right)$, l'âge et le poids d'abattage (Aaba, Paba) ainsi que les variables qui en découlent : GMQ durant les 60 premiers jours d'engraissement, entre $60 \mathrm{j}$ et l'abattage, sur l'ensemble de l'engraissement (GMQ60, GMQ6A, GMQEN), et la durée d'engraissement (Duen).

Les contrôles réalisés sur carcasses portent sur les points suivants : le poids carcasse (Pcar) est déterminé après $18 \mathrm{~h}$ de ressuage; le rendement carcasse ( $R d t)$ est approché par le rapport Pcar/Paba; la note de conformation (Nconf) est appréciée selon une échelle de 9 graduations comprises entre 1 et $5(E=5, U=4, R=3, O=$ 2, $P=1$ ). Sur les carcasses après $18 \mathrm{~h}$ de réfrigération, le jugement du niveau d'engraissement est réalisé à plusieurs endroits et donne lieu à notation selon 9 nuances étagées entre 1 et 5 : Ngext, Ngico estiment l'importance du recouvrement du gras externe et intercostal, Ngrog, celle du poids de gras interne périrénal (rognon). Une note de synthèse (Nsyn) est construite en pondérant Ngext, Ngrog, Ngico par les coefficients respectifs $1,0,5,0,5$. L'épaisseur du gras externe dorsal (Epgdo) est mesurée en $\mathrm{mm}$ après avoir pratiqué une fente au scalpel, latéralement aux $4^{e}$ et $5^{\mathrm{e}}$ vertèbres lombaires, à environ $2 \mathrm{~cm}$ de l'apophyse dorsale.

La note de tenue de gras (consistance, caractère huileux) est attribuée par un opérateur en fonction de 1 gradient allant du gras mou, huileux : note $=1$, au gras ferme et caillé : note $=5$. La note de couleur du gras externe est appréhendée, en référence à des nuanciers de couleur (références du catalogue Munsell) et organisée en 7 teintes croissantes dans un ordre de déclassement commercial : $1=$ blanc (réf $5 Y$. R 9/4), 2 = blanc rosé (réf 7.5 Y.R 9/4), 3 = rosé (réf 5 Y.R 8/8), 4 = rougeâtre (réf 7.5 Y.R 8/6), 5 = jaunâtre (réf 10 Y.R 9/4), 6 = rouge foncé (réf 7.5 Y.R 7/8), 7 = bistre avec reflets verdâtres (réf 10 Y.R 8/6), les classes $5,6,7$ pouvant être qualifiées de «bis".

Pour analyser l'influence des facteurs zootechniques sur la qualité de la tenue, 54 agneaux ont été choisis parmi les classes extrêmes de tenue ( 1 et 5 ). Afin de tenir compte des probables différences sexuelles, l'échantillon a été composé respectivement de $15 \%$ et 15 óen classe 1 , et de 14 get $100^{\prime}$ seulement, en 
classe 5 , en raison du manque de disponibilité en mâles bien classés sur ce critère. En effet, sur un ensemble de 1137 animaux contrôlés en abattoir, composé de $52,4 \%$ de mâles et de $47,6 \%$ de femelles, les classes extrêmes de tenue de gras apparaissaient à hauteur de 5,8\% pour les meilleures tenues (classe 5), respectivement $1 \%$ de of et $4,8 \%$ de $Q$ et de $14,3 \%$ pour les plus mauvaises tenues (classe 1 ), respectivement $10,4 \%$ de of et $3,9 \%$ de 8 .

\section{Composition des gras externes}

Pour tenter de mettre en relation les différences de nature des graisses sous-cutanées avec leurs propriétés de tenue après réfrigération, nous avons procédé à l'analyse d'un échantillon de tissu gras prélevé au niveau des vertèbres lombaires en position latéro-caudale. Ces prélèvements, broyés sous azote liquide à l'aide d'un broyeur à billes, furent conservés à $-18^{\circ} \mathrm{C}$ jusqu'à leur traitement.

L'extraction des lipides a été réalisée selon la méthode de Folch et al, 1957, en utilisant le mélange de solvants chloroforme/ méthanol (2/1 $\mathrm{v} / \mathrm{V}$ ) à raison de 3 fois $50 \mathrm{ml}$ pour $1 \mathrm{~g}$ de tissu gras, mixé à l'aide d'un homogénéiseur polytron. La phase chloroformique, après décantation en ampoule est déshydratée avec du sulfate de potassium anhydre, filtrée sur filtre Whatman séparateur de phase et conditionnée en plusieurs aliquots pour déterminer ultérieurement la teneur en lipides, l'indice d'acidité et la composition en acides gras (AG).

La teneur en lipides est obtenue par pesée après évaporation du solvant. L'indice d'acidité est déterminé selon la méthode AFNOR(NFV 03-906); celui-ci reste compris entre 0,4 et 1,2, confirmant la très faible proportion d'acides gras libres dans le tissu gras périphérique étudié. L'analyse de la composition en acides gras est réalisée, après préparation des esters méthyliques, selon la technique de transméthylation AFNOR(NFT 60-233), à l'aide d'un chromatographe Hewlett Packard 5890, sur colonne capillaire en silice fondue avec phase greffée PEG-TPA (FFAP) $(\varnothing=0,2 \mathrm{~mm}, L=50 \mathrm{~m})$. Le programme de montée en température appliqué est de $15^{\circ} \mathrm{C} / \mathrm{min}$ entre $150^{\circ} \mathrm{C}$ et $200^{\circ} \mathrm{C}$, de $5^{\circ} \mathrm{C} / \mathrm{min}$ entre $200^{\circ} \mathrm{C}$ et $210^{\circ} \mathrm{C}$, de $1,5^{\circ} \mathrm{C} / \mathrm{min}$ entre $210^{\circ} \mathrm{C}$ et $220^{\circ} \mathrm{C}$, avec des paliers à $200^{\circ} \mathrm{C}, 210^{\circ} \mathrm{C}, 220^{\circ} \mathrm{C}$ de $12 \mathrm{~min}, 5 \mathrm{~min}$ et 12 min respectivement, soit un temps d'analyse de 41 min pour un échantillon.

Au total, 30 agneaux ont servi à cette approche à raison de 15 dans chacune des classes de tenue, soit en classe $1: 7 \%$ et 8 ơ et en classe $5: 11$ q et $4 \%$. Ce déséquilibre résulte de la nécessité d'effectuer un prélèvement sanguin antérieurement à l'abattage et de la faible proportion de mâles classés en 5 .

\section{Paramètres sériques}

Une ponction a été réalisée dans la veine jugulaire, à l'aide de tubes venoject imprégnés d'héparinate de lithium. Les échantillons étaient placés aussitôt dans la glace avant séparation des plasmas dans un délai de moins de $4 \mathrm{~h}$. Les échantillons ont été conservés à $-18^{\circ} \mathrm{C}$ avant d'être soumis à l'analyse.

Les dosages hormonaux de la testostérone, de l'œstradiol, de la progestérone et de la thyroxine $\left(T_{4}\right)$ ont été effectués dans le but de vérifier le rôle probable de ces orientateurs métaboliques et les différences liées au sexe des animaux.

La cortisolémie a été mesurée pour mettre en évidence une éventuelle sensibilité au stress, mais aussi pour établir une liaison avec la progestéronémie. Le dosage de la Vit $\mathrm{B}_{12}$ a été réalisé pour examiner l'hypothèse d'une carence relative associée à un excès d'acide propionique. L'ensemble de ces dosages a été réalisé par le Laboratoire d'Hormonologie de l'ENV de Nantes à l'aide de la technique de dosage radioimmunologique (RIA) avec des kits Amersham prêts à l'emploi pour $T_{4}$, le cortisol, la progestérone, la Vit $\mathrm{B}_{12}$; bioMérieux pour l'œstradiol; $\mathrm{CIS}$ bioindustrie pour la testostérone.

\section{Analyse des résultats}

La confrontation des données de nature différente : paramètres d'élevage ante mortem, paramètres de carcasse, composition en acides gras du tissu sous-cutané, a été abordée par l'analyse des composantes principales (ACP). L'étude des paramètres les plus significatifs à l'égard du critère de tenue de carcasse est 
complétée par les comparaisons des moyennes (test de Student) et des corrélations associées à la composition et à la couleur du gras souscutané.

\section{RÉSULTATS}

\section{Composition du gras sous-cutané}

L'analyse en composantes principales conduite sur les différents acides gras constitutifs des triglycérides du tissu adipeux sous-cutané est résumée fig $1 a, b$. Les variables introduisant diverses combinaisons de ces acides gras sont positionnées en variables supplémentaires ainsi que les variables de notes de couleur et de tenue de carcasse.

Sur le plan $1 \times 2$ résumant $69 \%$ de l'inertie totale (tableau II), l'opposition sur le 1 er axe entre les variables MS, $\mathrm{C}_{18}, \mathrm{C}_{16}$ et l'ensemble des $A G$ ramifiés : $I_{15}$, $\mathrm{AIC}_{15}, \mathrm{IC}_{16}, \mathrm{IC}_{17}, \mathrm{AlC}_{17}, \mathrm{IC}_{18}$, et de $4 \mathrm{AG}$ mono-insaturés mineurs : $\mathrm{C}_{14}=1, \mathrm{C}_{15}=1$, $\mathrm{C}_{16=1}, \mathrm{C}_{17=1}$, est très nette. Sur le $2^{\mathrm{e}}$ axe, nous notons également une opposition très nette entre $C_{18=1}$ et $C_{14}$. Les variables supplémentaires $\mathrm{Nten}$ et $\mathrm{Ncou} /$ sont associées au premier axe. La fermeté des gras est étroitement corrélée aux fortes proportions de $\mathrm{C}_{18}$ et de MS, à l'inverse les couleurs foncées sont plutôt liées au deuxième ensemble d'AG mais de façon assez ténue.

La projection graphique des individus (fig 1b) discrimine très distinctement, sur ce même $1^{\text {er }}$ axe, les tenues fermes des gras mous et huileux. En revanche, cette analyse ne révèle pas de différence liée spécifiquement au sexe des agneaux. Enfin, les $A G: C_{14}, C_{18=1}, C_{18=2}, C_{18=3}$, et $\mathrm{C}_{20}$ ne semblent pas constituer des caractéristiques liées à la qualité des gras de carcasse.

A partir du tableau III regroupant les moyennes de composition du tissu gras selon les tenues, assorties des corrélations simples établies entre les pourcen-

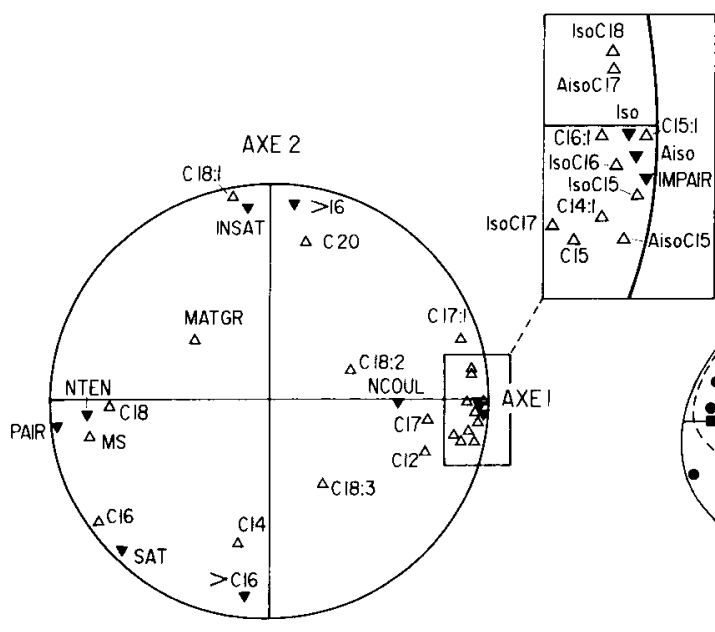

A

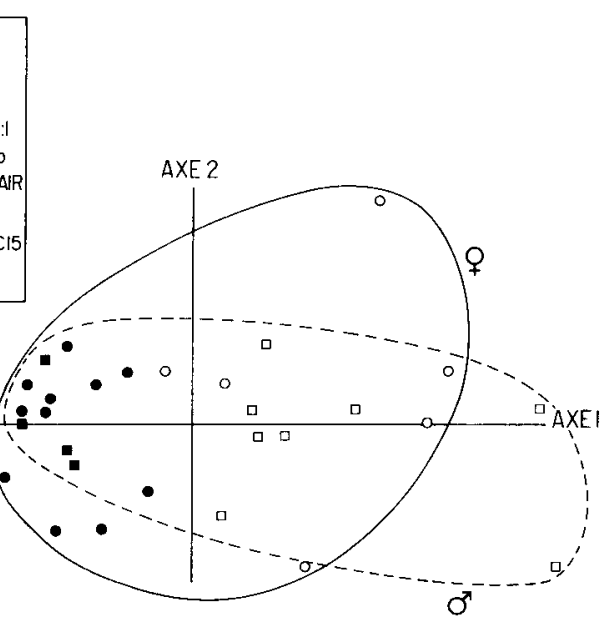

B

Fig 1. Analyse en composantes principales relative à la composition du tissu gras sous-cutané : projection sur le plan $1 \times 2$. a) Projection des variables : $\Delta$ var actives; $\boldsymbol{\Delta}$ var supplémentaires. b) Projection des individus : mauvaise tenue $\square\left(\sigma^{\circ}\right), O(o)$; bonne tenue $\mathbf{0}\left(\delta^{\circ}\right), \bullet(\ell)$. 
Tableau II. Analyse en composantes principales de la composition : contribution des 5 premiers axes à l'inertie totale.

\begin{tabular}{llllll}
\hline Axes & 1 & 2 & 3 & 4 & 5 \\
\hline $\begin{array}{l}\text { \% de linertie } \\
\text { Variance }\end{array}$ & $56,9 \%$ & $12,2 \%$ & $\begin{array}{l}9,4 \% \\
2,06\end{array}$ & $\begin{array}{l}5,1 \% \\
1,12\end{array}$ & $\begin{array}{l}4,6 \% \\
1,01\end{array}$ \\
\hline
\end{tabular}

Tableau III. Composition du tissu adipeux selon la note de tenue.

\begin{tabular}{|c|c|c|c|c|c|c|c|}
\hline \multirow[t]{2}{*}{ Composition } & \multirow[b]{2}{*}{$\begin{array}{c}\text { Tenue (1) } \\
(n=14)\end{array}$} & \multirow[b]{2}{*}{$\mathrm{CV} \%$} & \multirow[b]{2}{*}{$S^{*}$} & \multirow[b]{2}{*}{$\begin{array}{c}\text { Tenue (5) } \\
(n=15)\end{array}$} & \multirow[b]{2}{*}{ CV\% } & \multicolumn{2}{|c|}{ Corrélations simples } \\
\hline & & & & & & $\begin{array}{l}\text { Tenues } \\
(n=29)\end{array}$ & $\begin{array}{l}\text { Couleurs } \\
(n=29)\end{array}$ \\
\hline MS $\%$ poids frais & 74,2 & 4,5 & $* * *$ & 84,9 & 2,4 & 0,90 & $-0,50$ \\
\hline MG \% MS & 89,4 & 5,4 & NS & 92,3 & 4,0 & 0,33 & $-0,35$ \\
\hline $\mathrm{C}_{12} \%$ AG totaux & 0,3 & 51,5 & $\star \star \star \star$ & 0,1 & 50,2 & $-0,65$ & 0,31 \\
\hline $\mathrm{C}_{14}$ & 2,5 & 28,9 & NS & 2,8 & 28,1 & 0,16 & $-0,28$ \\
\hline$C_{14=1}^{14}$ & 2,0 & 23,8 & 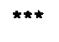 & 0,9 & 29,4 & $-0,83$ & 0,56 \\
\hline$i C_{15}^{14=1}$ & 0,4 & 42,2 & $* * *$ & 0,1 & 40,9 & $-0,77$ & 0,55 \\
\hline $\mathrm{aiC}_{15}^{\mathrm{b}}$ & 0,4 & 35,5 & $* * *$ & 0,2 & 27,0 & $-0,75$ & 0,50 \\
\hline $\mathrm{C}_{15}$ & 1,8 & 31,3 & $\star * *$ & 1,0 & 23,0 & $-0,72$ & 0,41 \\
\hline$C_{15=1}^{15}$ & 0,9 & 44,2 & $\star \star \star \star ~$ & 0,2 & 39,1 & $-0,79$ & 0,53 \\
\hline$i C_{16}$ & 0,6 & 52,2 & $\approx * *$ & 0,2 & 30,3 & $-0,67$ & 0,45 \\
\hline$C_{16}^{16}$ & 19,1 & 22,8 & 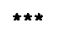 & 25,1 & 10,8 & 0,65 & $-0,45$ \\
\hline$C_{16=1}^{16}$ & 5,0 & 10,9 & $\star \star \star \star$ & 2,9 & 13,7 & $-0,92$ & 0,65 \\
\hline $\mathrm{iC}_{17}^{16}=1$ & 0,3 & 36,5 & $\star \star \star \star$ & 0,1 & 32,8 & $-0,68$ & 0,61 \\
\hline $\mathrm{aiC}_{17}$ & 1,7 & 24,4 & $* * *$ & 1,0 & 31,2 & $-0,72$ & 0,60 \\
\hline $\mathrm{C}_{17}$ & 3,1 & 22,6 & $\star \star \star$ & 2,4 & 26,3 & $-0,47$ & 0,45 \\
\hline$C_{17=1}$ & 4,6 & 27,0 & $\star \star * *$ & 2,0 & 22,5 & $-0,83$ & 0,52 \\
\hline$i_{18}$ & 0,7 & 45,8 & 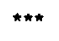 & 0,3 & 35,5 & $-0,68$ & 0,54 \\
\hline$C_{18}$ & 4,4 & 27,7 & $* * *$ & 9,0 & 24,0 & 0,80 & $-0,54$ \\
\hline$C_{18=1}^{18}$ & 49,2 & 7,0 & NS & 49,3 & 5,4 & 0,02 & $-0,03$ \\
\hline$C_{18=2}$ & 3,2 & 28,1 & NS & 2,8 & 23,5 & $-0,25$ & 0,38 \\
\hline$C_{18=3}$ & 0,3 & 19,9 & NS & 0,3 & 37,9 & $-0,01$ & 0,05 \\
\hline $\mathrm{C}_{20}$ & 0,3 & 53,4 & NS & 0,3 & 21,6 & $-0,16$ & $-0,16$ \\
\hline Sat & 35,7 & 12,0 & $* * *$ & 42,5 & 5,6 & 0,72 & $-0,50$ \\
\hline Insat & 64,9 & 5,0 & NS & 65,3 & 4,4 & 0,08 & $-0,01$ \\
\hline Pairs & 88,0 & 3,2 & $* * *$ & 94,0 & 13,4 & 0,82 & $-0,57$ \\
\hline Impairs & 13,2 & 22,3 & $\star * * *$ & 7,0 & 19,2 & $-0,82$ & 0,57 \\
\hline anteiso & 2,1 & 25,3 & $* \star \star *$ & 1,1 & 28,2 & $-0,75$ & 0,59 \\
\hline iso & 2,0 & 42,9 & $* * *$ & 0,7 & 29,9 & $-0,72$ & 0,54 \\
\hline Ramifiés (15 à 18) & 4,1 & 33,6 & $\star \star * \star$ & 1,9 & 28,2 & $-0,74$ & 0,57 \\
\hline $\begin{array}{l}\text { Mono-insat } \\
(14,15,16,17)\end{array}$ & 12,5 & 18,1 & $\star \star * *$ & 5,9 & 13,2 & $-0,90$ & 0,60 \\
\hline Sat $(16,18)$ & 23,5 & 20,4 & $* * *$ & 34,1 & 7,3 & 0,82 & $-0,56$ \\
\hline
\end{tabular}

$\mathrm{S}^{*}$ : signification à $P<5 \%: *, P<1 \%:{ }^{* *}, P<0,1 \%::^{* * *}$ (test de Student). 
tages d'acides gras et, respectivement, les notes de tenue et de coloration, nous constatons les éléments suivants :

- les gras mous sont caractérisés par une forte teneur en eau $(25,8 \%$ contre $15,1 \%)$ et des concentrations plus importantes $(+112 \%)$ en $\mathrm{C}_{14=1}, \mathrm{C}_{15=1}, \mathrm{C}_{16=1}$ et $\mathrm{C}_{17=1}$. Secondairement, les proportions en AG ramifiés sont aussi accrues (formes iso et anteiso). Enfin, la présence des AG impairs est nettement confirmée dans ce groupe (+ $89 \%)$.

- les gras fermes sont essentiellement associés à une forte représentation en $\mathrm{C}_{18}$ $(+104 \%)$ et plus globalement en AG saturés à nombre pair de carbones $\left(\mathrm{C}_{16}\right.$ et $\mathrm{C}_{18}$ ).

- les teneurs en $\mathrm{C}_{14}, \mathrm{C}_{18=1}$ n'ont pas d'influence sur ce critère de tenue.

- nous remarquons une incidence assez réduite des divers $A G$ constitutifs sur la coloration, avec cependant une liaison voisine de $r=0,60$ pour $\mathrm{C}_{16=1}, \mathrm{IC}_{17}$ et $\mathrm{AlC}_{17}$. En outre, des teneurs accentuées en $C_{18}$ et $\mathrm{C}_{16}(r=-0,57 ; r=-0,45)$ pourraient être associées à la blancheur du tissu gras.

Dans l'ensemble, la composition en AG explique bien la notion de tenue du gras sous-cutané, avec des corrélations élevées pour les AG influents, alors que les liaisons avec la note de coloration restent médiocres.

\section{Paramètres zootechniques}

L'analyse des données en ACP est réalisée selon 2 approches, afin de tenir compte de la nature différente des variables étudiées : une première confrontation résume le champ de variation des paramètres zootechniques ante mortem, les paramètres de carcasse étant introduits en variables supplémentaires, la seconde permet de préciser la situation inverse. 1er cas : observation des paramètres d'élevage (10 variables)

Les 3 premiers axes résument $83,2 \%$ de la variabilité totale (tableau IV). Le fer axe (fig 2a) est lié étroitement aux variables suivantes : $P e_{60}(r=-0,93)$, Gmqen ( $r=$ $-0,92), G m q_{60}(r=-0,89), G m q_{6} a\langle(r=$ $-0,75)$; il peut être assimilé aux performances de croissance. Le $2^{e}$ axe, de façon moins nette, montre une déformation du nuage selon une direction correspondant à des paramètres préétablis pour l'essentiel: Nais $(r=0,72)$, Aaba $(r=0,65)$, Paba $(r=0,60)$. Des Paba, distincts pour les 2 genres, ont été fixés par le protocole d'abattage et la projection des individus sur le plan $1 \times 2$ (fig $2 b$ ) discrimine très bien les 2 sexes d'agneaux, notamment au niveau du $2^{e}$ axe. Par rapport à l'axe de croissance, l'influence du sexe entraîne un décalage vers les plus faibles valeurs pour les femelles. Nous pouvons remarquer, en nous intéressant aux variables supplémentaires (fig 2a), une tendance pour la note de tenue à s'opposer aux performances de croissance sur le $1^{\text {er }}$ axe.

La tendance pour les ơ et les $q$ à posséder une carcasse molle pour les performances de croissance élevées, et une consistance ferme pour les valeurs les plus faibles est nette (fig 2b), mais nous observons là encore, un décalage entre les 2 sexes.

Nous remarquons par ailleurs quelques exceptions bien tranchées. Ainsi, 2 o" sont classés en mauvaise tenue, malgré une faible vitesse de croissance : il s'agit en fait d'individus issus de naissances multiples ( 3 et 4 agneaux). II en est de même pour 2 femelles et un mâle possédant à la fois une forte vitesse de croissance et une bonne qualité de carcasse : ils proviennent d'un même élevage qui pratique une complémentation sous la mère avant sevrage. 
Tableau IV. Analyse en composantes principales des paramètres $z$ contribution des 5 premiers axes à l'inertie totale.

\begin{tabular}{llllll}
\hline \multicolumn{1}{c}{ Axes } & 1 & 2 & 3 & 4 & 5 \\
\hline $\begin{array}{l}\text { Paramètres d'élevage } \\
\text { - \% de l'inertie } \\
\text { - Variance }\end{array}$ & & & & & \\
Paramètres de carcasse & $46 \%$ & $24,4 \%$ & $12,8 \%$ & $9,4 \%$ & $4,3 \%$ \\
$\begin{array}{l}\text { - \% de l'inertie } \\
\text { - Variance }\end{array}$ & 4,60 & 2,44 & 1,28 & 0,94 & 0,43 \\
& $23 \%$ & $19,5 \%$ & $14,6 \%$ & $11,5 \%$ & $9,6 \%$ \\
\hline
\end{tabular}

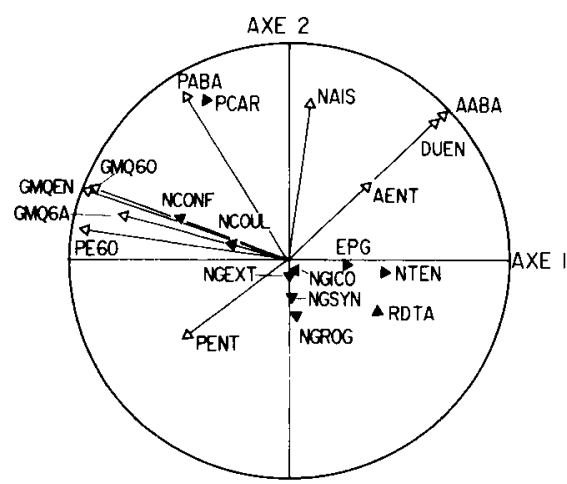

A

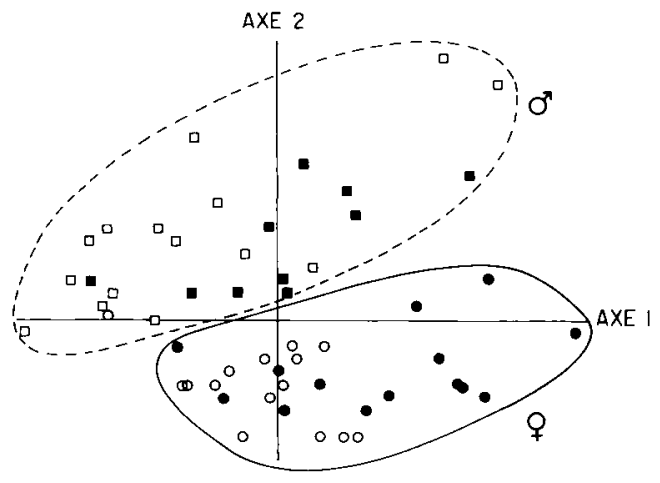

B

Fig 2. Analyse en composantes principales relative aux paramètres d'élevage : projection sur le plan $1 \times 2$. a) Projection des variables : $\Delta$ var actives; $\Delta$ var supplémentaires. b) Projection des individus : mauvaise tenue $\square\left(\sigma^{\prime}\right) ; O(q)$; bonne tenue $\left(\sigma^{\prime}\right), 0(\%)$.

\section{$2^{e}$ cas : Observation des paramètres de carcasse (10 variables)}

Les relations entre les paramètres de carcasse sont moins affirmées, les 4 premiers axes principaux ne contribuant que pour $68,6 \%$ à l'inertie totale (tableau IV). L'axe 1 (fig 3a) est plutôt représentatif des notes de gras Ngext et Ngsyn ( $r=-0,64, r=-$ $0,75)$, l'axe 2 de $\mathrm{Ngico}(r=0,60)$ et assez faiblement de Nten $(r=48)$ et de Ncoul $(r=$ $-0,58)$; l'axe 3 est lié majoritairement à $\operatorname{Nconf}(r=0,59)$ et Pcar $(r=0,64)$, mais de façon assez peu représentative.
Sur le plan de projection constitué par les axes 1 et 2, résumant $42,5 \%$ de l'inertie totale, 3 groupes de variables s'individualisent pouvant être interprétés comme :

g.1 : la tenue et la quantité de gras;

g.2 : la conformation et la couleur foncée des carcasses;

g.3 : le recouvrement adipeux de la carcasse.

Les 2 premiers regroupements s'opposent, le troisième restant indépendant des 2 autres dans cette vue. 

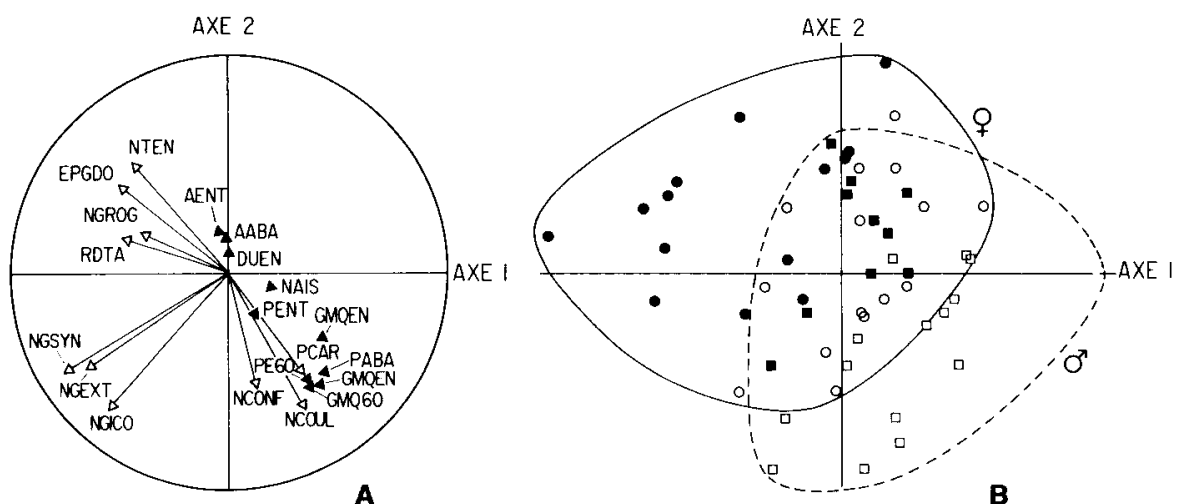

Fig 3. Analyse en composantes principales relative aux paramètres de carcasse : projection sur le plan $1 \times 2$. a) Projection des variables : $\Delta$ var actives; $\boldsymbol{\Delta}$ var supplémentaires. $b)$ Projection des individus : mauvaise tenue $\square\left(\sigma^{\top}\right), O(q)$; bonne tenue $\boldsymbol{\omega}(\delta), \bullet(q)$.

La répartition des points individus sur le plan 1,2 (fig 3b) indique une moins bonne discrimination des sexes que dans le premier cas. Femelles de mauvaise qualité et mâles de bonne qualité sont confondus. Seules les extrémités : mâles à gras mou et femelles à gras ferme sont nettement opposées. En fait, l'axe secondaire constitué par les projections des variables $P$ car et Nten est discriminant à cet égard.

En conséquence, il convient d'analyser séparément $\delta$ et $q$ qui se distinguent nettement, tant pour les variables zootechniques d'élevage, que pour celles concernant les carcasses.

Le tableau IV de comparaison des moyennes confirme ces premières indications en laissant apparaître quelques différences en rapport avec le facteur tenue de carcasse.

\section{Chez les mâles}

Les agneaux possédant une bonne tenue sont caractérisés par un Gmq moindre sur l'ensemble de la période d'engraissement ( $324 \mathrm{~g}$ contre $368 \mathrm{~g}$ ); leur couverture de graisse sous-cutanée est plus épaisse
( $4 \mathrm{~mm}$ contre $2 \mathrm{~mm}$ ) et leur coloration de graisse est claire (2,6 contre 4,5). De même l'Aent semble supérieur $(P<0,10)$ pour ces animaux ( $35 \mathrm{j}$ contre $31 \mathrm{j})$.

\section{Chez les femelles}

Nous retrouvons des écarts comparables pour le Gmqen (264 g contre $307 \mathrm{~g}$ ) et qui restent significatifs entre $60 \mathrm{j}$ et l'abattage $\left(G m q_{6} a: 282 \mathrm{~g}\right.$ contre $327 \mathrm{~g}$ ), ainsi qu'une nuance de couleur semblable. S'ajoutent des différences significatives pour $P e_{60}$ Duen et naturellement Aaba $(P<0,001)$. Par ailleurs, les agnelles ayant une bonne tenue de carcasse apparaissent plus grasses, comme le révèle la note de gras de synthèse NGSYN (3,5 contre 2,7).

L'analyse conjointe des corrélations (tableau IV) montre que les tendances à l'égard de la tenue de carcasse sont assez comparables pour les variables les plus influentes déjà signalées, en restant cependant moins accentuées chez les agneaux mâles. Spécifiquement, pour les agnelles, Pent et Nten sont liées négativement, Nconf et Nten, positivement, alors que chez les agneaux 0 ces variables restent 
Tableau V. Comparaison des paramètres zootechniques dans le cas des agneaux mâles et femelles présentant des caractéristiques extrêmes de tenue de gras sous-cutanés.

\begin{tabular}{|c|c|c|c|c|c|}
\hline Variables d'élevage & \multirow[b]{2}{*}{ Mâles } & \multirow{2}{*}{\multicolumn{2}{|c|}{ Femelles }} & \multicolumn{2}{|c|}{ Corrélations simples } \\
\hline & & & & Tenue & Couleur \\
\hline $\begin{array}{c}\text { Tenue } 1 \\
\text { (gras mou, } \\
\text { huileux) } \\
(\mathrm{n}=15) \text { CV\% }\end{array}$ & $\begin{array}{c}\text { Tenue } 5 \\
\text { (gras ferme, } \\
\text { caillé) } \\
(n=10) \text { CV\% }\end{array}$ & Tenue 1 & Tenue 5 & & \\
\hline
\end{tabular}

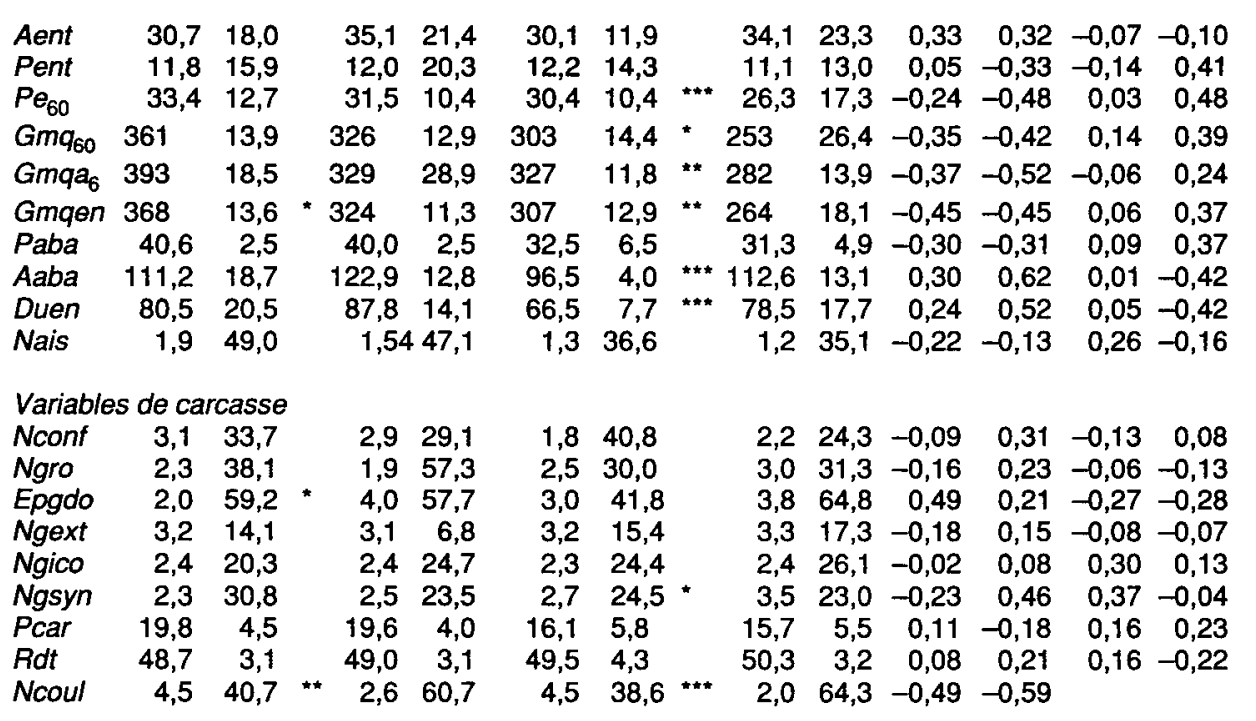

$\mathrm{S}^{*}$ : signification à $P<5 \%: *$ à $P<1 \%: " *$ a a $P<0,1 \%: * *$ (test de Student).

indépendantes. Enfin, nous enregistrons une tendance de l'âge au sevrage (Aent) a être corrélé positivement à la note de tenue chez les 2 sexes $(P<0,10)$.

\section{Paramètres sériques}

Sexes analysés séparément, aucune différence significative n'apparaît pour les divers paramètres plasmatiques contrôlés (Test, Estr, Prog, Cort, Thyr, Vit $\mathrm{B}_{12}$, tableau VI). II est donc difficile d'invoquer une éventuelle influence de ces paramètres, sans doute en raison de la forte variabilité de nos résultats et de la faible représentativité de nos échantillons, notamment pour la composante mâle avec une bonne tenue.

Si ce n'est pour l'écart de teneur évident en testostérone entre of et $q$, il n'apparaît pas non plus de différence convaincante entre sexes. En conséquence, l'influence de ces facteurs sur la qualité des réserves lipidiques n'apparaît pas pour cet échantillon.

Sur quelques animaux, la comparaison des valeurs sériques contrôlées en élevage et au moment de l'abattage montre 
Tableau VI. Comparaison des paramètres sériques en fonction de la tenue.

\begin{tabular}{|c|c|c|c|c|c|c|c|c|c|c|}
\hline \multirow{3}{*}{$\begin{array}{l}\text { Paramètres } \\
\text { sériques }\end{array}$} & \multicolumn{5}{|c|}{ Mâles } & \multicolumn{5}{|c|}{ Femelles } \\
\hline & \multicolumn{2}{|c|}{ Tenue 1} & \multirow[t]{2}{*}{$S^{*}$} & \multicolumn{2}{|c|}{ Tenue 5} & \multicolumn{2}{|c|}{ Tenue 1} & \multirow{2}{*}{$S^{*}$} & \multicolumn{2}{|c|}{ Tenue 5} \\
\hline & $(n=10)$ & CV\% & & $(n=4)$ & $\mathrm{CV} \%$ & $(n=7)$ & CV\% & & $(n=10)$ & CV\% \\
\hline Test pmol/l & 1897 & 136,3 & NS & 3267 & 115,1 & 187 & 264,5 & NS & 0 & \\
\hline Oestr pmol/ & 68 & 46,8 & NS & 66 & 54,0 & 70 & 16,8 & NS & 117 & 96,0 \\
\hline Prog nmol/l & 1,5 & 56,7 & NS & 1,2 & 40,0 & 1 & 58,0 & NS & 1,3 & 89,2 \\
\hline Cort nmol/I & 111 & 50,8 & NS & 79 & 62,7 & 116 & 60,6 & NS & 123 & 41,1 \\
\hline Thyr pmol/l & 33 & 20,0 & NS & 32 & 57,3 & 34 & 27,2 & NS & 37 & 55,4 \\
\hline$\vee B_{12} \quad \mathrm{ng} / \mathrm{l}$ & 1,6 & 33,7 & NS & 1,7 & 49,7 & 1,8 & 28,8 & NS & 1,4 & 38,7 \\
\hline
\end{tabular}

$S^{*}:$ signification à $P<0,05$ (test de Student).

une certaine stabilité des teneurs en thyroxine, en Vit $B_{12}$ et en progestérone. A l'inverse, la situation de stress et/ou de décalage horaire modifient considérablement les teneurs en œstrogène, cortisol et testostérone.

\section{DISCUSSION}

L'analyse en composantes principales fait ressortir que l'influence des sexes est nettement marquée pour les variables d'élevage. Sur les paramètres de carcasse, celle-ci devient moindre et elle n'intervient plus sur la composition du tissu gras étudié dans notre échantillon.

\section{Composition du tissu gras sous-cutané et qualité}

Comme l'ont signalé de nombreux auteurs (Garton et al, 1972; Molénat et Thériez, 1973; Miller et al, 1980; Aurousseau, 1981), le caractère mou du tissu gras sous-cutané est à mettre en relation avec le point de fusion de ses triglycérides constitutifs. Les acides gras insaturés et/ou ramifiés sont à ce titre responsables de l'aspect huileux des carcasses qui ne parviennent pas à cailler convenablement lors de leur conservation en chambre froide à $4{ }^{\circ} \mathrm{C}$. La trame conjonctive ne joue certainement qu'un rôle secondaire car nous retrouvons la même distinction : gras durs, gras mous, très nettement confirmée, pour des échantillons préalablement broyés finement et congelés, donc aux structures cellulaires éclatées, lors de leur réchauffement à la température ambiante.

La tenue des gras de couverture est aussi étroitement associée à la teneur en eau. S'agit-il d'une moindre maturité des adipocytes non encore envahis complètement par les inclusions lipidiques ou bien d'une hydratation particulière des tissus périphériques? Les résultats de Jenkins et Thonney, 1988 indiquent que chez les agneaux à fort niveau d'ingestion, les proportions de lipides et d'eau corporels augmentent, comparativement à un plus faible niveau de consommation, ceci indépendamment de la concentration énergétique 
alimentaire et de la propionémie. Un mécanisme antidiurétique pourrait-il accompagner l'hyperphagie ? Ferrell et al (1986) constatent qu'à l'occasion d'une forte croissance compensatrice, le métabolisme de base des agneaux est augmenté et que, corrélativement, la composition du croît s'accompagne d'une réduction de la proportion de lipides et d'un accroissement de la teneur en eau.

Pour ce type d'agneau sevré et engraissé en bergerie, les différences entre classes extrêmes de carcasses (1 contre 5) sont associées à un déséquilibre des rapports d'acides gras saturés/insaturés $(0,55$ contre 0,65$)$, d'une part, et d'acides gras impairs ramifiés/pairs $(0,19$ contre 0,09 ), d'autre part, comme l'ont déjà montré plusieurs auteurs (Field et al, 1978; Aurousseau, 1981; Busboom et al, 1981).

Plus précisément, nous notons une forte proportion d'acides gras à chaîne moyenne $\left(C_{14}\right.$ à $\left.C_{17}\right)$ mono-insaturée. En revanche, la proportion d'acide oléique $\left(C_{18=1}\right)$ reste indépendante du caractère tenue de gras. Une forte représentativité (environ $50 \%$ ) et son point de fusion intermédiaire $\left(13,4^{\circ} \mathrm{C}\right)$ en font un agent neutre dans le phénomène de caillage en chambre frigorifique. Nous ne notons pas non plus de différences en rapport avec la proportion des $A G$ longs polyinsaturés, d'ailleurs peu représentés.

Les $A G$ à courte chaîne $C_{12}$ à $C_{15}$, qui vraisemblablement ont une orgine microbienne (Church, 1979a), en particulier pour les acides à nombre impair de carbones, sont également caractéristiques des gras mous. Cette composition du tissu gras laisse supposer l'intervention de plusieurs mécanismes possibles car l'origine des précurseurs de ces acides gras à nombre impair de carbones et/ou ramifiés est variée.
L'utilisation, à des fins énergétiques, de l'isoleucine et des dérivés antéiso de la valine et de la leucine (Church, 1979b) par le métabolisme microbien dans le rumen, peut conduire à des amorces impaires d'acides gras. Dans ce cas, ces acides gras bactériens peuvent être captés par la cellule adipeuse après leur absorption intestinale.

L'autre source d'acides gras impairs et ou ramifiés est associée aux fortes concentrations, rumenales et sériques, en propionate. Sous la forme propionyl-CoA, celui-ci peut être utilisé comme initiateur de la chaîne d'acide gras, à la place de l'acétate, dans la synthèse de novo par le complexe acide gras synthétase, aboutissant aux acides gras à nombre impair de carbones. II peut aussi s'intégrer dans la chaîne carbonée par le biais du méthyimalonate $\mathrm{CoA}$, produisant alors des acides gras ramifiés (Church, 1979b; Busboom et al, 1981; Aurousseau, 1986; Guesnet et Demarne, 1987). L'implication d'un défaut relatif en $V_{i t} B_{12}$ a été proposée pour expliquer l'insuffisance de la cellule hépatique à transformer l'acide méthylmalonique en malonyl-CoA (Cardinale et al, 1970; Aurousseau, 1981). Nous n'observons pas de différence au sein de notre échantillon dans la vitaminémie de nos animaux; une carence ne peut être évoquée, les niveaux rencontrés $(>1,4 \mathrm{ng} / \mathrm{l})$ étant proches de la normale (O'Harte et al, 1989). D'ailleurs, les essais de supplémentation n'apportent guère d'amélioration (Duncan et al, 1974). Une carence relative peut-elle suffire à expliquer cette déviation?

Une autre cause d'accumulation de propionyl-CoA peut résider dans la compétition des diverses formes de précurseurs glucogéniques. Aiello et al, 1989 démontrent que le butyrate possède une action antagoniste spécifique sur la transforma- 
tion hépatique du propionate en glucose. Or, la proportion de butyrate s'accroît lorsque l'offre alimentaire ou le bas $\mathrm{pH}$ rumenal favorise son passage intestinal (Annison, 1984; Aiello et al, 1989) aux dépens de la forme hydroxylée.

\section{Incidence des facteurs zootechniques}

La confrontation des paramètres d'élevage et des caractéristiques de carcasses permet de suggérer quelques hypothèses.

La vitesse de croissance enregistrée sur l'ensemble de l'engraissement est corrélée négativement, comme tous les paramètres pondéraux, à la fermeté du gras de couverture. En plus d'une production importante d'acide propionique, liée à la nature du concentré distribué, la forte ingestion accompagnant les vitesses de croissance élevées implique conjointement une activité métabolique intense, favorable à l'anabolisme protidique. Dans ce contexte, la restérification intraadipocytaire peut se réaliser préférentiellement à partir des acides gras insaturés, les acides gras saturés étant remis en circulation en plus grandes proportions (Leat, 1977; Aurousseau, 1981), à condition que la lipolyse ne soit pas trop intense.

Chez les femelles, et de façon moins prononcée chez les mâles, contrairement aux indicateurs de vitesse de croissance, nous retrouvons des liaisons positives entre tenues fermes et les paramètres zootechniques associés à un degré de maturité plus avancé (Duen, Aaba, Ngsyn). S'agissant de l'influence des sexes et des délais d'engraissement, les conclusions de Crouse et al, 1978, sont ici clairement confirmées. En revanche, dans le cas d'un même régime alimentaire, et pour les 2 sexes, la précocité nous paraît être à l'inverse des performances de crois- sance pondérales, dès lors que les comparaisons s'établissent à poids constant.

Les différences entre sexes sont d'autant plus accentuées que le protocole d'abattage retenu pour nos animaux, à partir d'un poids de 30 et $39 \mathrm{~kg}$, respectivement pour $\%$ et $\sigma^{\prime}$, confère un degré de maturité plus avancé pour les agnelles (43\% contre $39 \%$ ). Nous expliquons la moindre qualité des mâles, en général, par un poids d'abattage ne correspondant pas à une maturité suffisante, compte tenu de leur potentiel génétique de croissance. La différence significative de l'Epgdo enregistrée chez les mâles pour les qualités de tenue 1 et $5(2 \mathrm{~mm}$ contre $4 \mathrm{~mm})$ abonde dans ce sens.

Il est probable que la qualité des dépôts sous-cutanés soit liée au degré de maturité des adipocytes, les formes juvéniles ou âgées étant plus sujettes à la constitution de triglycérides chargés en acides gras insaturés. Au contraire, en phase d'accrétion maximale, les acides gras saturés prédominent en raison de la réduction de la lipolyse et de la captation majorée des acides gras issus des triglycérides circulants d'origine alimentaire. En outre, Church, 1979b, relate que la lipogenèse de novo élabore des triglycérides avec des acides gras en plus grande proportion désaturés que lorsque ceux-ci sont synthétisés à partir des ac gras exogènes. Ce phénomène est d'ailleurs dépendant de l'âge, puisque la part de la synthèse de novo représente $25 \%$ de la lipogenèse entre 6 et 12 mois, alors qu'elle n'est que de $10 \%$ entre 5 et 7 mois. Une réduction relative de cette synthèse de novo pourrait faciliter l'obtention de gras fermes.

Dès lors, il semblerait souhaitable de prolonger l'engraissement des animaux les moins matures, en acceptant d'alourdir les carcasses. Dans le cas de cette race, cette catégorie correspond aux agneaux qui 
possèdent les performances de croît les plus élevées, surtout chez les mâles. Si les poids d'abattage doivent être maintenus pour des raisons commerciales, une sélection des géniteurs sur la précocité serait favorable.

Quelles que soient les options, la qualité des lipides alimentaires du régime de fin d'engraissement est certainement déterminante, puisque ceux-ci contribuent beaucoup, durant cette phase, à la constitution du tissu gras. Solomon et Lynch, 1988, attribuent d'ailleurs leur résultat concernant une plus grande fermeté des gras souscutanés des agneaux nourris avec le régime le plus énergétique de leurs essais, aux fortes proportions de saindoux introduites dans l'aliment. II serait donc envisageable d'adapter la qualité de l'aliment de finition au potentiel génétique des agneaux de bergerie.

L'augmentation de l'âge au sevrage (Aent), pour les 2 sexes, tend à améliorer la qualité du gras $(P<0,10)$. La durée d'allaitement peut conditionner, en effet, la nature des gras développés ultérieurement par les agneaux (Molénat et al, 1971; Molénat et Thériez, 1972; Thériez et al, 1973). II conviendrait sans doute de prolonger l'effet bénéfique des lipides constitutifs du lait maternel sur la composition des gras de carcasse (Aurousseau et al, 1973). L'aliment de transition entre le sevrage et la phase d'engraissement est donc à considérer, en particulier sous l'angle de la qualité des lipides introduits. L'emploi d'AG à courte chaîne carbonée, inférieure à $\mathrm{C}_{16}$, non seulement favorise la digestibilité des lipides, mais surtout permet l'utilisation de ce substrat énergétique dans des conditions optimales. Un effet immédiat serait de freiner la lipolyse consécutive au sevrage durant la phase d'adaptation à un nouveau mode d'alimentation. Cette orientation métabolique permet, en outre, une épargne protéique (Asplund et al, 1985), réduisant les risques de formation d'ac gras ramifiés à partir de l'utilisation énergétique des ac aminés. Cette conjonction, pour des animaux encore proches du stade monogastrique, est intéressante pour répondre à l'extériorisation du potentiel de croissance durant cette période d'anabolisme protidique intense (Touliec et Quillet, 1987; Cera et al, 1989).

\section{CONCLUSION}

De l'ensemble des observations réalisées sur cet échantillon de cas extrêmes en matière de tenue de gras de carcasse, après ressuage et réfrigération, il ressort, aussi bien pour les mâles que pour les femelles, que les individus exprimant une forte vitesse de croissance sont sujets à produire des carcasses à la tenue médiocre et, à un moindre degré, au développement de colorations intempestives. Le gras sous-cutané de mauvaise qualité se distingue par une composition enrichie surtout en acides gras mono-insaturés moyens $\left(C_{14}=0\right.$ à $C_{17}$ $=0$ ) et une plus forte teneur en eau. Cependant, les rythmes de croissance des 2 sexes sont différents, les femelles, plus précoces, atteignent un degré d'engraissement supérieur à celui des mâles au poids d'abattage retenu, respectivement 30 et 39 kg. Dès lors, il semblerait approprié de conduire le rationnement des agneaux spécifiquement selon leur sexe et en fonction de leur potentiel génétique de croissance. Par ailleurs, l'influence positive de la durée d'engraissement sur la qualité de carcasse semble établir un effet de l'âge, et sans doute, du stade de maturité du tissu adipeux.

Des manipulations alimentaires pourraient modifier cette fragilité des agneaux les plus performants en matière de vitesse de croissance. Dès le début de l'engraissement, la limitation du déficit énergétique 
alimentaire de la phase de post-sevrage avec l'emploi de lipides dotés en acides gras courts à moyens est à considérer. En fin d'engraissement, il serait opportun, dans le cas du maintien d'un poids constant à l'abattage pour satisfaire à une argumentation commerciale, de repérer les individus à forte vitesse de croissance à partir d'un critère de maturité exprimé en pourcentage du poids à l'état adulte, et d'accélérer leur adipogenèse en leur réservant un aliment enrichi en acides gras longs, appropriés.

Si l'alourdissement des carcasses ne constitue pas un handicap majeur, la prolongation de la durée d'engraissement serait un autre atout favorable.

Enfin, une action de sélection génétique serait certainement fondée compte tenu des fortes héritabilités sur les critères d'engraissement (Bouix et al, 1986). Cependant, cette sélection, liée à la précocité, amènerait certainement à raccourcir le format des béliers retenus (Baird et al, 1989) et serait en opposition avec la recherche d'une vitesse de croissance élevée, des carcasses lourdes et peu grasses. La dualité dans les objectifs de production qui visent un niveau d'engraissement faible dans la classification Europa, mais avec un gras de bonne qualité, amène à penser qu'il reste indispensable d'établir une adéquation entre régime alimentaire et potentialités génétiques. Nous rejoignons là les conclusions principales de Field et al, 1978.

\section{RÉFÉRENCES}

Aiello RJ, Armentano LE, Bertics SJ, Murphy AT (1989) Volatile fatty acid uptake and propionate metabolism in ruminant hepatocytes. J Dairy Sci 72, 942-949
Annison EF (1984) The metabolism of neutral and acidic lipids by tissues of the ruminant. In: Herbivore Nutrition in Subtropics and Tropics (Gilchrist FM, Mackie RI, eds) The Science Press Pub, Craighall, South Africa 549-570

Asplund JM, Ørskov ER, Hovell FDD, Mac Leod NA (1985) The effect of intragastric infusion of glucose, lipids or acetate on fasting nitrogen excretion and blood metabolites in sheep. Br J Nutr 54, 189-195

Aurousseau B (1981) Élaboration des lipides corporels et valeur des carcasses des ruminants. Bull Tech CRVZ Theix INRA 45, 43-50

Aurousseau B (1986) Influence des facteurs d'élevage sur l'état d'engraissement et la qualité des carcasses chez les bovins. In: Qualités des produits chez les ovins et les caprins. $11^{\theta}$ Journée rech Ovine et Caprine INRA-ITOVIC, SPEOC, Paris, 210-234

Aurousseau B, Thériez M, Daniel M (1973) Influence de la nature des matières grasses dans l'aliment d'allaitement sur le métabolisme lipidique de l'agneau de boucherie. Ann Biol Anim Biochim Biophys 13, 93-105

Baird RL, Tatum JD, Butler JG, Fitch GQ, Gilmore G (1989) Identification of optimal slaughter weight for small, medium and large framed wethers. Am Dairy Sci Abst 67-72 suppl, 202

Bouix J, Bibe B, Lefèvre C, Eychenne F (1986) Variabilité génétique entre et intrarace de la croissance et les qualités des carcasses d'agneaux. In: 11e Journée de la recherche ovine et caprine INRA, ITOVIC SPEOC, Paris, 115-125

Busboom JR, Miller GJ, Field RA, Crouse JD, Riley ML, Nelms GE, Ferrell CL (1981) Characteristics of fat from heavy ram and wether lambs. J Anim Sci 52, 83-92

Cardinale GJ, Carty TJ, Abel ES (1970) Effect of methylmalonyl coenzyme $A$, a metabolite which accumulates in vitamin $B_{12}$ deficiency, on fatty acid synthesis. $J$ Biol Chem 245, 3771-3776

Cera KR, Mahan DC, Reinhart CA (1989a) Apparent fat digestibilities and performance responses of post weaning swine fed diets sup- 
plemented with coconut oil, corn oil or tallow. $J$ Anim Sci 67, 2040-2047

Cera KR, Mahan DC, Reinhart CA (1989b) Postweaning swine performances and serum profile responses to supplemental medium chain free acids and tallow. J Anim Sci 67, 20482055

Church DC (1979a) Rumen metabolism of lipids. In: Digestive Physiology and Nutrition of Ruminants. Vol I, Oxford Press, Portland, Oregon, 253-265

Church DC (1979b) Lipid utilization and requirement. In: Digestive Physiology and Nutrition of Ruminants. Vol II : Nutrition. Oxford Press, Pub, Portland, Oregon, 167-186

Crouse JD, Field RA, Chant JL, Ferrell CL, Smith GM, Harrison VL (1978) Effect of dietary energy intake on carcass composition and palatability of different weight carcasses from ewe and ram lambs. $J$ Anim Sci 47, 12071218

Duncan WRH, Ørskov ER, Fraser C, Garton GA (1974) Effect of processing of dietary barley and of supplementary cobalt and cyanocobalamin on the fatty acid composition of lamb triglycerides, with special reference to branched chain components. Br J Nutr 32, 71-75

Elliot JM (1980) Propionate metabolism and vitamin $B_{12}$. In: Digestive Physiology and Metabolism in Ruminants (Ruckebush $\mathrm{Y}$, Thivend $P$, eds) MTP Press, Lancaster, 485-503

Ferrell CL, Koong LJ, Nienaber JA (1986) Effect of previous nutrition on body composition and maintenance energy costs of growing lambs. Br J Nutr 56, 595-605

Field RA, Williams JC, Ferrell CL, Crouse JD, Kunsman JE (1978) Dietary alteration of palatability and fatty acid in meat from light and heavy weight ram lambs. J Anim Sci 47, 858864

Folch J, Lees M, Sloane-Stancey CHS (1957) A simple method for the isolation and purification of total lipids from animal tissues. $J$ Biol Chem 226, 497-509

Garton GA, Hovell FD, Duncan WRH (1972) Influence of dietary volatile fatty acids on the fatty acid composition of lamb triglycerides with special reference to the effect of propionate on the presence of branched chain components. Brit J Nutr 28, 409-416
Girard JP, Randriamanarivo M, Denoyer C (1986) Les lipides animaux dans la filière viande. Vol II. Rôle des lipides dans le déterminisme des qualités de carcasse, de la viande, du tissu adipeux et des produits adipeux. Stn rech viande (INRA, Theix, ed) APRIA, Paris

Guesnet $P$, Demarne $Y$ (1987) La régulation de la lipogenèse et de la lipolyse chez les mammifères. INRA, Paris

Jenkins TC, Thonney ML (1988) Effect of propionate level in a volatile fatty acid salt mixture fed to lambs on weight gain, body composition and plasma metabolites. J Anim Sci 66, 1028-1035

Leat WHF (1977) Depot fatty acids of Aberdeen Angus and Friesan cattle reared on hay and barley diets. J Agric Sci Camb 89, 575-582

Miller GJ, Kunsman JE, Field RA (1980) Characteristics of soft subcutaneous fat in ram lambs fed corn and corn-silage diets. J Food Sci 45,279

Molénat G, Thériez M (1972) Allaitement artificiel des agneaux. II. Influence de la teneur en matières grasses des laits de remplacement. Ann Zootech 21, 285-299

Molénat G, Thériez M (1973) Influence du mode d'élevage sur la qualité de carcasse de l'agneau de bergerie. Ann Zootech 22, 279293

Molénat G, Thériez M, Aguer D (1971) L'allaitement artificiel des agneaux. I. Détermination de l'âge minimal au sevrage pour la production d'agneaux de boucherie. Ann Zootech 20, 339-352

Moody WG, Kemp JD, Mahyuddin M, Johnston DM, Ely DG (1980) Effect of feeding systems, slaughter weight and sex on histological properties of lamb carcasses. J Anim Sci $50,248-256$

O'Harte MFP, Kennedy DG, Blanchflower WJ, Rice DA (1989) Methylmalonic acid in the diagnosis of cobalt deficiency in barley fed lambs. Brit J Nutr 62, 729-738

Ørskov ER, Duncan WR, Carnie CA (1975) Cereal processing and food utilization by sheep. III. Effect of replacement whole barley by whole oats on food utilization and firmness and composition of subcutaneous fat. Anim Prod 21, 51-59 
Paruelle JL, Pain JM (1982) Alimentation et qualités des graisses de dépôt des carcasses d'agneaux de bergerie. Bull Tech UCAAB 1, 3-10

Solomon MB, Lynch GP (1988) Biochemical, histochemical and palatability characteristics of young ram lambs as affected by diet and electrical stimulation. J Anim Sci 66, 19551962

Thériez M, Molénat G, Danie! $M$, Aurousseau B (1973) L'allaitement artificiel des agneaux. ill. Influence de la nature des matières grasses incorporées dans l'aliment d'allaitement. Ann Zootech 22, 185-197
Toullec R, Quillet J (1987) Utilisation des lipides par le veau de boucherie. In: Lipides et alimentation animales évolution et perspectives. Colloque INAPG, Paris, 45-48

Van Quackebeke E, Cazes JP, Vallade C (1978) Alimentation et qualité des gras. In: Alimentation de la brebis et de la chèvre. $4^{\ominus}$ Journée Rech ovine et caprine. INRAITOVIC, SPEOC, Paris, 295-311

Vigneron $P$, Prud'hon $M$, Touraille $C$, Valin $C$, Bouix J, Bibe B (1986) Croissance des agneaux-Types de fibres musculaires et qualité de la viande-Muscles indicateurs. $\ln : 11^{\circ}$ Journée Rech ovine et caprine INRAITOVIC, SPEOC, Paris, 49-73 